\title{
Lexikografie der Kollokationen zwischen Anforderungen der Theorie und der Praxis
}

\begin{abstract}
Kollokationen stellen einen noch zu wenig beachteten Teilbereich der Phraseologie dar. Sie sind bisher in den Wörterbüchern unzureichend erfasst und werden auch nicht systematisch gelehrt und gelernt. Es lassen sich zwei Typen von Kollokationen unterscheiden, die sowohl eine strukturelle als auch eine statistische Herleitung nutzen und beide für die unauffällige und kompetente Sprachproduktion im Alltag von Bedeutung sind. Angesichts der großen Zahl der auffindbaren Kollokationen ist zu differenzieren und zu gewichten: a) Es steht lexikografisch zunächst die Unterstützung bei der Sprachproduktion im Vordergrund sowie b) der Grundwortschatz bzw. Basiswortschatz und es sind c) die Unterscheidung von typischen Wortverbindungen und gebräuchlichen Wortverbindungen (den Hund loslassen vs. den Hund anleinen/ an die Leine nehmen) vorzunehmen.
\end{abstract}

\section{Einführung}

\subsection{Ausgangslage}

Kollokationen sind in der Sprachwissenschaft inzwischen sehr präsent, nicht jedoch außerhalb des engeren wissenschaftlichen Umfeldes. Wer an einem Kollokationswörterbuch arbeitet, muss in seinem weiteren sozialen Umfeld fast täglich erklären, worum es sich dabei handelt.

In den Lehrmitteln und im Schulunterricht werden Kollokationen kaum behandelt, obwohl sie - wenn man die Größe des Bereichs, die unsichere Sprachpraxis sowie den Lehr- und Lernbedarf mit Bezug auf Kollokationen nicht nur im Zweitspracherwerb, sondern auch im Erstspracherwerb berücksichtigt - einen prominenten Stellenwert haben müssten (vgl. Burger 2010, S. 52).

Dabei ist es intuitiv immer einsichtiger, mit Kollokationen zu argumentieren, die weder in sich variiert werden können, noch alternative Ausdrucksmöglichkeiten kennen, die man also „richtig“ verwenden muss, um keinen Fehler zu machen: *eine Entscheidung nehmen (vs. die korrekte Form eine Entscheidung fällen bzw. treffen): Wortverbindungen dieser Art sind in einer bestimmten Form bzw. in bestimmten Varianten einer Form gebräuchlich (und in einer anderen Form falsch). Als nicht oder schwach idiomatische Wortverbindungen gehören sie unzweifelhaft zu den festen Wortverbindungen im phraseologischen Sinn (vgl. ebd., S. 11). 
Kollokationen einer Sprache, wie „leise Töne“ „Abschied nehmen“, ,zu vorgerückter Stunde“, ,schweren Herzens“, ,in Strömen regnen“, sind ein fester und in der Regel nicht aktiv wahrgenommener Bestandteil der alltäglichen Kommunikation. Sie werden beim Sprechen bzw. Schreiben nicht ad hoc gebildet, sondern als zusammengehörige Syntagmen abgerufen. Das hat zur Folge, dass alternative, frei kombinierte Konstruktionen (beispielsweise ,ruhige Töne") als unpassend empfunden werden, obwohl sie semantisch und syntaktisch ebenso korrekt wären.

Es gibt aber auch weitere Kollokationstypen, die weniger vor dem Hintergrund der sprachlichen Norm und der Fehlervermeidung als im Rahmen der Stilistik als bevorzugte Ausdrucksweisen identifizierbar sind: Es sind typische Wortverbindungen, die über die Ermittlung von Kookkurrenzen gefunden werden, die sich nicht einfach aus den Selektionsrestriktionen der Semantik der Wörter ergeben (wie die Kombination von Holz und brennen in einem Satz das Holz brennt, bei dem Brennbarkeit zu den Merkmalen von Holz gehört). Für die Textproduktion sind die typischen Kollokationen ebenso hilfreich. Beide Arten von Kollokationen bilden die usuellen Wortverbindungen im Sinne von Kathrin Steyer passim (vgl. Steyer/ Brunner 2009).

Kollokationen ...

a) sind ebenso wie idiomatische Phraseologismen auf der zeichentheoretischen Ebene von Wortverbindungen interessant;

b) haben sprachgeschichtlich mit der Ökonomie der Sprachproduktion zu tun (vgl. von Polenz 2009). Die Ausbildung von Kollokationen stellt für die Sprachproduktion eine Formulierungserleichterung durch Vorprägung dar und geschieht durch die Einengung der Kombinationsmöglichkeiten;

c) können textgeschichtlich als Musterentwicklungen erfasst werden, wobei „Muster“ sowohl die durch den Sprachgebrauch erreichte Vorgeprägtheit von Wortkombinationen als auch die Struktur von Wortverbindungen im Sinne einer Konstruktion meint (Zwillingsformel als Struktur, Funktionsverbgefüge als Struktur etc.);

d) haben mit Wissensorganisation und Wissensstrukturierung zu tun (vgl. Filatkina/Hanauska i.Dr.);

e) bilden sich ontogenetisch in der Erstsprache als feste Einheiten heraus, werden aber nicht notwendigerweise so gelernt, sondern können auch als vermeintlich produzierbare Wortverbindungen erworben werden (vgl. Häcki Buhofer 1997). Sie werden jedoch in der Zweitsprache oft bewusst und in bewusstem Kontrast von der Erstsprache gelernt (vor allem für den Erwerb des Englischen in Form von Listen von „phrasal verbs“ üblich). 


\subsection{Hintergrund: Forschungsprojekte}

Die vorliegende Auseinandersetzung ist vor dem Hintergrund von verschiedenen Forschungsprojekten entstanden, die in verschieden ausgerichteten lexikografischen Projekten, zum Teil auch Datenbankprojekten, Kollokationen erfassen, nämlich:

- „Wörterbuch der festen Wendungen und Wortverbindungen der deutschen Sprache“ (www.kollokationenwoerterbuch.ch) (Prof. Dr. Harald Burger, Prof. Dr. Brigit Eriksson, Dr. Hans Bickel, M.A. Marcel Dräger, lic. phil. Tobias Roth);

- „Das Neue Baseldeutsch-Wörterbuch“ (www.baseldeutsch.ch) (Dr. Lorenz Hofer, Dr. Markus Gasser, lic. phil. Emilie Buri, Stefanie Meier);

- „DWDS - Schweizer Textkorpus“ (www.chtk.unibas.ch) (Dr. Lorenz Hofer, Dr. Markus Gasser, lic. phil Tobias Roth, lic. phil. Christoph Schön);

- „Hyperhamlet“ (www.hyperhamlet.unibas.ch) (M.A. Sixta Quassdorf);

- Pro*Doc „Sprache als soziale und kulturelle Praxis“, Forschungsmodul „Typisierte Sprache - in Geschichte, Diskurs und Ontogenese“ (Doktoranden u.a. Andreas Bürki); „Zum Erwerb von Kollokationen“ (Qualifikationsarbeit: Stefanie Meier).

Die folgenden Ausführungen bewegen sich insbesondere vor dem Hintergrund des Projektes „Wörterbuch der festen Wendungen und Wortverbindungen der deutschen Sprache“ bzw. abgekürzt „Kollokationenwörterbuch".

Wir gehen von einer Lemmabasis von 2.000 Wörtern des Grundwortschatzes aus, die den Rahmen des Wörterbuches bilden sollen. Dafür haben wir einige Grund- und Lernwortschätze des Deutschen ausgewertet und mit den Frequenzlisten auf dem DWDS Schweizer Textkorpus, dem Wortschatzportal Leipzig und dem Deutschen Referenz-Korpus in Mannheim verglichen. Dazu wurde das Variantenwörterbuch ausgewertet. Gerade bei den im Vergleich zu Idiomen unauffälligen Kollokationen scheinen viele regionale oder nationale Varianten zu existieren. Zu den 2.000 Wörtern des Grundwortschatzes werden in den oben genannten Korpora die kookkurrenten Wortverbindungen gesucht und daraus potenzielle Kollokationen, die im Kontext des Alltags nützlich sein können, ausgewählt. Nun gibt es natürlich nicht zu jedem Lemma des Grundwortschatzes eine Kollokation, dafür jedoch zu einigen Lemmata mehrere Kollokationen. Am häufigsten sind Substantive an Kollokationen beteiligt; sie machen im Basiswortschatz etwa $2 / 3$ aus. Sie sind also wichtig, aber man kann sich nicht auf Substantivkombinationen beschränken. Dafür werden u.a. vor dem Hintergrund des Sprachvergleichs Komposita berücksichtigt, wo man es in anderen Sprachen mit Kollokationen zu tun hat. 


\subsection{Thesen}

Die systematische lexikografische Erfassung und Beschreibung von deutschen Kollokationen steht noch aus, auch wenn die Wörterbücher der deutschen Sprache, insbesondere das Stilwörterbuch des Dudenverlags, viele Kollokationen als Lemmavarianten in Beispielsätzen und Paraphrasen - ohne spezifische Kennzeichnung - enthalten.

Eine separate Lexikografie der Kollokationen der deutschen Standardsprache wird zeigen, dass systematische Korpusanalysen der Standardsprache auf der Basis von Kookkurrenzanalysen viel zusätzliches Material zu Tage fördern.

Bezüglich der Festigkeit von Kollokationen ist die Unterscheidung von gebräuchlichen und typischen Wortverbindungen sinnvoll: den Hund anleinen/an die Leine nebmen (normative Varianten) vs. den Hund loslassen (typische Formulierung, stilistisch erfassbar). Mit der Benennung der unterschiedlichen Typen von Kollokationen kann man sich schwer tun, die unterschiedlichen Kriterien können jedoch aufgeführt werden und stellen normative Gebräuchlichkeit, die die Falschheit von Alternativen impliziert, und häufige Ausdrucksweise im Sinne von typischer Üblichkeit gegeneinander.

Bei der Arbeit im Rahmen der deutschschweizerischen Dialektlexikografie lassen sich auch zusätzliche Kollokationen finden, die jedoch - wohl in erster Linie der relativ kleinen Korpora wegen - quantitativ weniger ausgeprägt sind. Hier sind ergänzende Datenerhebungen über Umfragen hilfreich.

Die bisherige Lexikografie von Kollokationen insgesamt - das gilt für Standardsprachwörterbücher und Dialektwörterbücher gleichermaßen - verzeichnet Kollokationen, wenn überhaupt - ohne metasprachliche Kennzeichnung in Beispielsätzen. Auf diese Weise wird den BenutzerInnen weder klar, dass es sich dabei um Kollokationen handelt, noch können - ohne Angabe einer Nennform - die Möglichkeiten der formalen Verwendungen beurteilt werden. Es ist aber wünschenswert, dass die BenutzerInnen von Wörterbüchern auch über Kollokationen informiert werden: Kollokationen sind grundsätzlich nicht nur im Hinblick auf die Sprachrichtigkeit lexikografisch zu erfassen, sondern auch im Hinblick auf die Ausbildung von Sprachwissen lexikografisch angemessen darzustellen.

\subsection{Definitionen}

Kollokationen stellen im deutschsprachigen Forschungsbereich einen Teilbereich der Phraseologie dar (vgl. Burger 2010, S. 57). Als prototypisch gelten bei Kollokationen Substantiv-Verb-Verbindungen mit normativ festgelegtem Verb: Anzeige erstatten (nicht Anzeige stellen o.Ä.). Die Bandbreite der 
Strukturtypen ist jedoch viel größer und reicht von eine Rolle spielen bis zu vielfältigen adverbialen Kollokationen wie in Kürze, in diesem Augenblick oder aus persönlichen Gründen. Kollokationen werden grundsätzlich eher von der normativen Gebräuchlichkeit, der Üblichkeit und Usualität, der Typizität her als von der Semantik aus definiert. In semantischer Hinsicht gelten Kollokationen als „normal“, transparent, weil vollmotiviert wie freudiges Ereignis, eventuell auch als teilmotiviert (wie der blinde Passagier) (vgl. Barz 2007, S. 27; Burger 2010, S. 38 und S. 70). Die Üblichkeit wird oft durch die Häufigkeit des Miteinandervorkommens der Teile bestimmt, so beim DWDS, wo es auf der Titelseite der Homepage heißt - „Wortverbindungen, die besonders häufig gemeinsam auftreten“, wobei gemeint ist, deren Teile bzw. Elemente besonders häufig miteinander auftreten. Man kann zwei Operationalisierungsrichtungen der Kollokationsdefinition unterscheiden, nämlich die Korpuslinguistische Operationalisierung vs. Fehlerlinguistische Operationalisierung. Die Korpuslinguistische Operationalisierung sucht Kookkurrenzen in Korpora mit Hilfe mathematischer Verfahren und scheidet Wortkombinationen aus, die keine semantisch sinnvollen Wortverbindungen darstellen (wie ,und der“, die zwar häufig nacheinander vorkommen, aber semantisch keine Wortverbindung bilden). Die Fehlerlinguistische Operationalisierung geht von Varianten aus, die als fehlerhaft empfunden bzw. eingestuft werden (müssen). Diesen falschen Gebrauchsweisen, die auch in Texten wie Zeitungsartikeln häufig vorkommen, welche von professionellen Schreibenden verfasst werden, werden die richtigen Varianten gegenübergestellt. Die Fehlerlinguistische Operationalisierung kann ergänzt werden durch die Überlegung, wie eine Übersetzung in eine andere bekannte Sprache lauten müsste: Wenn sie nicht Wort für Wort erfolgen kann, ist die Wahrscheinlichkeit, dass eine Kollokation vorliegt, relativ hoch. Tener admiracion kann man mit Bewunderung haben (wörtlich) übersetzen, besser aber mit Bewunderung hegen, was es laut DWDS (bzw. seiner Kollokationsberechnung vom 12.01.2010) als Kollokation nicht gibt, wo man Bewunderung nur erregt. Bewnuderung hegen ist hingegen laut Wörterbuch der Deutschen Gegenwartssprache (WDG) möglich. Die Kollokationsberechnung ergibt hingegen Wünsche, Befürchtungen, Zweifel, die man hegt. Entsprechende Abklärungen sind oft durch Unvollständigkeiten aus der Korpusabfrage ebenso wie der lexikografischen Verzeichnung erschwert.

Varianten zu Kollokationen sind falsch im Sinne der Sprachnorm, z.B. in Fällen wie *eine Entscheidung nehmen oder *eine Notiz lassen oder *eine Anzeige stellen (vgl. „Die XY-Immobilien AG bedauert den Vorgang und stellt als betroffene Eigentümerin Strafanzeige." Aus: Neue Zürcher Zeitung, 10.02.2010, S. 17). Anzeige muss man erstatten. Die automatische Kollokationenanzeige aus dem DWDS zeigt für Anzeige, dass erstatten zum Kollokationengefüge gehört, neben ganæseitig etc., und dass erstatten zu Anzeige 
führt, daneben auch zu Bericht und Meldung etc., die man auch noch erstatten kann. Stellen bringt demgegenüber bei der DWDS-Kollokationenanzeige feststellen, eine Frage stellen oder zur Verfügung stellen und bildet einen häufigen Kollokator, aber eben nicht den richtigen, wenn es um die Anzeige geht.

Man spielt eine Rolle, man gibt sie oder macht sie nicht, auch wenn man die Ophelia geben kann. Diese Fälle sind vergleichsweise einfach, die über das Fehlerempfinden operationalisiert werden können. Sie entstehen ebenso häufig bei L1 SprecherInnen, die nicht die richtige Verbindung finden bzw. ein Verbindungselement aus einem anderen Kontext verwenden, als auch bei L2 SprecherInnen, die eine Kollokation aus der Erstsprache übertragen und damit die Kollokation für native speakers erst so richtig deutlich machen. Schaden hingegen muss man nicht unbedingt anricbten, auch wenn das eine unauffällig professionelle Ausdrucksweise ist, man kann ihn auch verursachen.

Kookkurrenzen, also statistisch festgestellte feste Wortverbindungen (verschieden berechenbar, beispielsweise entsprechend Steyer/Brunner 2009, S. 2) bilden ein Reservoir für Kollokationen, sind aber nicht samt und sonders Kollokationen in einem lexikogrammatischen normativen oder stilistischen Sinn (siehe unten). Kollokationen sind gemäß ihrem Charakter als Wortverbindung jedenfalls nicht als beliebige bzw. als „freie Wortverbindungen“ einzustufen. Diese Nicht-Beliebigkeit besteht allenfalls nur in einer Vorprägung bzw. Formelhaftigkeit im nicht-semantischen Sinn.

$\mathrm{Ob}$ man solche kollokativen Mehrworteinheiten „Formeln“ nennen soll, ist eine offene Frage. Laut dem Handbuch zur Sprach- und Kommunikationswissenschaft zur Phraseologie (Burger et al. (Hg.) 2007) bilden Kollokationen zusammen mit Idiomen als Phraseme nur den engeren Bereich der Phraseologie. Es wird empfohlen, bei einer weiten Konzeption auf andere Termini wie formelhafte Sprache oder Texte oder feste Wortverbindungen „,auszuweichen“ (ebd., S. 3). Kollokationen können Routineformeln enthalten oder auch nicht (ebd., S. 4). Es besteht auch die Möglichkeit, Routineformeln als kommunikative Phraseologismen von referenziellen Phraseologismen abzutrennen, die ihrerseits als nominative Phraseologismen in Kollokationen, Teil-Idiome und Idiome aufgeteilt werden können (vgl. Burger 2010, S. 36 f.).

Referenzielle Phraseologismen beziehen sich auf Objekte, Vorgänge oder Sachverhalte der Wirklichkeit, während kommunikative Phraseologismen bestimmte Aufgaben bei der Herstellung, Definition, dem Vollzug und der Beendigung kommunikativer Handlungen haben. Referenzielle Phraseologismen können als satzgliedwertige Phraseologismen (im Unterschied zu den satzwertigen) semantisch untergliedert werden: als Kollokationen, TeilIdiome und Idiome sind sie im Wesentlichen semantisch bestimmt.

Man macht nicht notwendigerweise einen sprachlichen Fehler, wenn man sich „nicht-kollokativ“ ausdrückt, aber es tönt oft nicht professionell für den Empfänger und ist keine ökonomische Ausdrucksweise, mit der 
man sich aus dem Wortverbindungsreservoir und -repertoire bedient. Kollokationen sind in der festen Form in der Alltagskommunikation unauffällig und unmarkiert und - was ebenso wichtig ist - in der fachnahen oder fachinternen Kommunikation für den Eindruck der Professionalität wesentlich. Als fachnahe Kommunikation müssen weite Bereiche der Alltagskommunikation verstanden werden, in der beispielsweise medizinische, psychologische, schulische oder administrative Dinge besprochen werden, ohne dass die Beteiligten als Fachleute ausgebildet sind. Weil Kollokationen unauffällig und unmarkiert sind, ist es oft schwierig, sie sich bzw. Anderen bewusst zu machen. Jmdn. mit Verdacht auf einen Herzinfarkt ins Spital einliefern ist die professionelle Ausdrucksweise, die eine verbale und eine präpositionale Kollokation enthält. Wer davon spricht, dass ein Bekannter ins Spital gebracht werden musste, weil der Arzt vermutete, dass er einen Herzinfarkt gehabt haben könnte - die kollokative Ausdrucksweise wäre einen Her乏infarkt erlitten haben könnte - wird genauso verstanden, drückt sich aber mit freien Paraphrasen, oft etwas länger, oft weniger genau im professionellen Sinne oder einfach weniger professionell aus.

Während Kollokationen in Schulbüchern und im Schulunterricht eine gewisse Präsenz und Aufmerksamkeit erfahren, liegen Kollokationen der Erstsprache (den Tisch decken, die Nase putzen) unter der Aufmerksamkeitsund Bewusstseinsschwelle.

Franz Josef Hausmann hat das basisbezogene Kollokationskonzept eingeführt (Hausmann 2004, S. 321, vgl. auch 1989, S. 1010). Dabei geht er in vielen diskutierten Fällen von einer möglichen Unterscheidung richtig/ falsch in Bezug auf die Substantiv-Verb-Verbindung aus. Danach stellt sich wesentlich das Problem, welches Verb korrekterweise zu einem bestimmten Substantiv gehört. Das Substantiv ist die Basis, das Verb der Kollokator (Abscbied nehmen). Nach diesem Konzept sind Basisartikel im Wörterbuch gefragt, Kollokatorenartikel im Wörterbuch jedoch überflüssig. Wenn und weil die Kombinationen Substantiv-Verb arbiträr und unvorhersehbar sind, soll, um der Ausdrucksnot zu begegnen, ein Ausdruckswörterbuch bzw. ein Produktionswörterbuch geschaffen werden.

Kollokatorenartikel können jedoch ebenso hilfreich sein wie Artikel zum (oft substantivischen) Basiswort. Dafür sollen vier Gründe angeführt werden:

1) Die Richtung, in der eine passende Ergänzung gesucht wird, ist nicht immer eindeutig und man merkt oft, wenn man sich überlegt, ob eine Wortverbindung gebräuchlich oder typisch ist, dass man nicht vom Substantiv her argumentiert: So kann der Hund mit allen möglichen Verben kombiniert werden, aber wedeln bzw. mit dem Schwanz wedeln wird typischerweise von einem Hund gesagt. 
2) Auch syntaktisch komplexere Wortverbindungen bilden Kollokationen, wie beispielsweise Substantiv-Verbindungen unter Einbezug von Präpositionen wie vom Stubl (berunter) fallen, Substantiv-Adjektivverbindungen (mit oder ohne Präposition) wie leise Töne, werdende Mutter, schweren Herzens, bimmelweiter Unterschied, zu vorgerückter Stunde. Sie bilden Strukturen, die je nach grammatischer Ausrichtung auf einer Skala von grammatisch vielseitig variabel bis formelhaft oder in typischen Ausdrucksweisen vorgeprägt angeordnet werden können (vgl. Eichinger 2004: Skala „Redewendungen bis zu syntaktischen Schematisierungen“). Dazu kommt schließlich, dass für die Unterstützung der Entwicklung von Sprachwissen auch Kollokatorenartikel hilfreich sind.

3) Relativ allgemeine Verben mit vielfältiger und wenig spezifizierter Semantik wie stellen, legen, setzen, machen etc. können sehr viele Basiswörter „bedienen“: stellen hat vergleichsweise diejenige breite Palette von möglichen Verbindungen, die man im Auge hat, wenn man sagt, dass es „,nichts bringt", einen Artikel zu stellen (zu verfassen) („,̈̈berflüssige Kollokatorenartikel“, vgl. Hausmann 2004, S. 312). Wenn man jedoch ein Wort wie stellen hat und sich fragt, was man damit noch so „machen“ kann, hat auch diese Fragerichtung ihre Berechtigung, allerdings weniger für die Hausmannsche „Momo-Situation“, in der jemand schon ein Wort hat und die angemessene Ergänzung sucht, als für Fragen des Sprachwissens.

4) Zudem gibt es auch eine recht große Zahl seltener, veraltender oder unikaler Kollokatoren, die man wegen ihrer spezifischen Gebrauchsweise als Lemmata aufführen sollte, beispielsweise bei jmdm. das Leben vergällen; vergällen steht auf einer Vorstufe der Unikalität, indem das Verb fast nur noch gebunden an Freude oder eben Leben vorkommt und sollte entsprechend - je nach Häufigkeit der Wortverbindung als Ganzer und Ausrichtung des Wörterbuches - als eigenes Lemma aufgeführt werden; ebenso kommt bezichtigen mit wenigen Substantiven wie Lüge, Untreue oder Plagiat vor.

Die Heterogenität der verbalen Kollokatoren stellt bereits einen Hinweis darauf dar, dass alle Bestandteile von Kollokationen in die Analyse einzubeziehen sind und lexikografisch adäquat zu behandeln sind.

Eine Sprachtheorie muss nicht nur erklären, wie man aus wenigen syntaktischen Regeln praktisch unendlichen Nutzen ziehen kann,

sondern auch, wie uns Kollokationen und Kookkurrenzen helfen, Strukturen auf der lexikalischen Ebene schneller und effizienter zu durchdringen. Wenn lexikalische Einheiten nicht völlig unabhängig voneinander auftreten, bedeutet das einen wesentlichen Schritt zur Reduktion von Komplexität: wir werden auf Erwartbares, in diesem Sinn Normales verwiesen. (Eichinger 2004, S. 5) 
Bei Eichinger werden Kollokationen im Kontext wiederholter Rede gesehen: Für die Lernerlexikografie, aber auch für die Lexikografie überhaupt ist die Darstellung von wiederholter Rede, die nicht unmittelbar grammatische Regularitäten betrifft, aber die für die Akzeptabilität von Äußerungen zentral ist, eine wichtige Aufgabe (ebd., S. 5.). Auch für die Professionalität des Sprechens sind Kollokationen wichtig - und zwar nicht nur die berufliche, sondern auch die Alltagsprofessionalität, die ja mehr und mehr auch von Fachdiskursen mitgeprägt ist.

Als Phänomene wiederholter Rede gehören Kollokationen in den Kontext der historischen und ontogenetischen Musterentwicklung und des Musterlernens - dazu wird in Basel und Zürich an einigen Dissertationen gearbeitet (im Rahmen des Pro*Doc-Forschungsmoduls „Typisierte Sprache - in Geschichte, Diskurs und Ontogenese"), die Muster nicht als passive Imitationsübung, sondern als Einheiten der sprachgeschichtlichen Entwicklung im Kontext aktiver kognitiver Organisations- und Strukturierungsform sehen. Andreas Bürki (i.Dr.) untersucht am Schweizer Textkorpus Mehrworteinheiten, zu denen die Kollokationen gehören, und stellt die Entwicklung, sprachgeschichtliche Prägung und Varianz von Mehrworteinheiten in Jahrhundertvierteln dar.

Die folgende Tragetaschen-Werbung macht Gebrauch von einem solchen Muster, indem potenzielle Nichtraucher wie zukünftige Mütter durch den identischen Musterteil „für werdende $X_{\text {“ }}$ auf Coop Lebensmittel verwiesen werden, die als Alternative zu Zigaretten genossen werden können.



Abb. 1: Werbung „Für werdende Mütter“



Abb. 2: „Für werdende Nichtraucher“ 


\section{Lexikografische Situation und Methoden der Darstellung und Auffindung}

\subsection{Problematik der lexikografischen Darstellung von Kollokationen}

Es gibt einen beachtlichen Stand der Lexikografie von Kollokationen, wie er im Duden 2 zur Stilistik und in anderen Wörterbüchern eher implizit, für das Englische im Oxford Dictionary zu „collocations“ explizit realisiert ist. Kollokationen haben aber in der deutschsprachigen Lexikografie noch keinen festen Platz.

Es ist nicht klar, wie die Kollokationen, die bisher in Wörterbüchern behandelt wurden, ermittelt wurden; ob statistische Verfahren, die bloße Häufigkeit, Autorenintuition oder vielleicht doch Kriterien der Festigkeit eine Rolle spielen. Sie stehen im Wörterbuch häufig ohne metakommunikative Kennzeichnung als typische Verwendungen, als Phrasen oder als Beispielformulierungen, seltener als Lemmata (vgl. Steyer 2008; Steyer/Brunner 2009 sowie Burger 2010).

Den Wörterbuchbenutzern auf jeden Fall wird nicht klar, was Kollokationen und was einfach typische Beispiele für Wortverwendungen sind. Das gilt auch für VerfasserInnen von Wörterbüchern, wenn sie als NutzerInnen versuchen, diese Wörterbuchartikel zu analysieren.

\subsection{Vergleichender Ertrag systematischer Korpusabfragen}

Die begonnenen Arbeiten zeigen schon bisher: Korpora liefern viel zusätzliches Material. Einerseits erhält man über Kookkurrenzanalysen (neben gröBeren Mengen auszusortierenden Materials) Belege mit ergänzenden Formen oder anderem Schwerpunkt als aus dem Wörterbuch hervorzugehen scheinen. Die Abfragen stellen also Bewertungshilfen dar. Andererseits liefern Korpora aber auch bisher lexikografisch nicht oder allenfalls als Beispielsätze verzeichnete Kollokationen.

Korpusabfragen sind aber - wie wir auch aus anderen Zusammenhängen wissen - keine Allheilmittel für alle lexikografischen Fragen, die man an den Sprachgebrauch hat. Manchmal ergeben sie auch einfach „nichts“, obwohl man als kompetenter Sprecher oder kompetente Sprecherin sozusagen sicher ist, dass eine bestimmte Ausdrucksweise fest vorgeprägt, usuell, gebräuchlich oder typisch ist. Das ist an einem Vergleich der Abfragen beim DWDS (www.dwds.de) mit dem Stilwörterbuch des Duden Verlags (2010) konkret an einzelnen Beispielen zu zeigen.

Wenn man den Vergleich Wörterbuch - Korpus quantitativ abzuhandeln versucht, was zugegebenermaßen einige Probleme der Operationalisierung der Zählung der Nennungen mit sich bringt, so kann das Resultat in der Größenordnung ungefähr und mit Vorsicht zu genießen etwa folgender- 
maßen aussehen, wobei eine solche im Wörterbuch gezählte Nennung beispielsweise lauten kann: streunender Hund. Die Korpusanalyse bringt beispielsweise zusätzlich bissiger Hund.

\begin{tabular}{|l|c|c|c|}
\hline & Schnittmenge & Stilwörterbuch & Korpusanalyse \\
\hline Hund & 16 & 16 & 46 \\
\hline Wetter & 32 & 37 & 60 \\
\hline Ehre & 26 & 62 & 33 \\
\hline Laune & 11 & 14 & 15 \\
\hline Summe & 85 & 129 & 154 \\
\hline
\end{tabular}

Tab. 1: Anzahl der Kollokationen mit Hund, Wetter, Ehre und Laune im Vergleich Duden Stilwörterbuch - DWDS

Man ersieht aus diesem versuchsweisen quantitativen Vergleich (Tab. 1), dass es jeweils große Schnittmengen der Kollokationen zu Hund, Wetter, Ehre und Laune gibt. Die Kookkurrenzanalyse in einem Korpus fördert zusätzliche Kollokationen zu Tage, findet aber auch nicht alle Kollokationen, die das Stilwörterbuch verzeichnet. Die Differenzen sind zu erwarten, die Ergebnisse der Kookkurrenzanalysen aus angemessen großen und systematisch aufgebauten Korpora jedoch unbedingt einzubeziehen.

Im Folgenden werden drei Wörterbuchartikel zu den Lemmata Hund, Laune und Ehre aus dem Duden Stilwörterbuch aufgeführt und diskutiert. Die in diesen Artikeln aufgeführten Kollokatoren zu den jeweiligen Lemmata wurden um weitere, durch Korpusrecherchen im DWDS ermittelte Kollokatoren ergänzt. Die korpusbasiert ermittelten Kollokatoren sind mit einem Pfeil markiert. Dabei wird keine Aussage darüber gemacht, ob die mit Pfeil markierten Lemmata nicht eigenständig oder andernorts aufgeführt sind.

Das Stilwörterbuch spricht von „festen Verbindungen und Wendungen“ und meint damit alle Phraseologismen (vgl. Duden 2010, S. 11 f.). Die „festen Wortverbindungen und Wendungen" stehen im Druck abgehoben (farbig gedruckt) und mit einem Sternchen versehen am Ende des Artikels. Es werden folgende Arten unterschieden:

1) Feste Attribuierungen: schwarzer Markt,

2) Feste Verbindungen: einen zwitschern,

3) Funktionsverbgefüge: zur Verteilung kommen/gelangen,

4) Wortpaare (Zwillingsformeln): ab und zu; bei Nacbt und Nebel,

5) Feste Wendungen: etw. auf die lange Bank schieben. 
Die jeweilige Verbindung oder Wendung ist beim (ersten) Substantiv oder, wenn kein Substantiv vorkommt, beim ersten sinntragenden Wort aufgeführt (ebd.).

„Wörter, die nur in einer festen Verbindung oder in bestimmten Wendungen vorkommen, werden in der ihnen eigenen Form der Verwendung vorgeführt" (Duden 2010, S. 13):

z.B. ausfindig: (in der Verbindung) jmdn., etw. ausfindig machen


stehen.

\section{Hund, der}

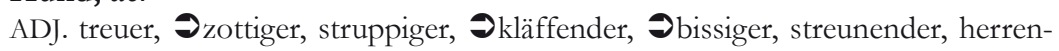
loser

SUBST. $\rightarrow$ Hund und Katze

H. (subj.) + VERB der H. bellt, Oेbellt jmdn. an, kläfft Kleine Hunde kläffen häufiger als große. schlägt an, winselt Nachbars Hund war alleine und winselte den ganzen Abend. jault, heult, $\boldsymbol{\nabla}$ knurrt, $\boldsymbol{O}$ fletscht, $\boldsymbol{O}$ schnappt, beisst (jmdn.) Beisst ihr Hund? Nein, der ist ganz brav. | $\boldsymbol{\partial}$ der H. beisst jmdn. in etw. Erst fletschte der Hund die Zähne, dann biss er dem Postboten in die Hose. wedelt mit dem Schwanz, Ohechelt, Orisst Mein Hund frisst am liebsten Wurst. $\boldsymbol{O}_{\text {schnüffelt | }}$ der H. wittert eine Fährte, $\boldsymbol{\vartheta}$ jagt, $\boldsymbol{O}$ holt ein Stöckchen/einen Ball/... Immer und immer wieder bolte der Hund das Stöckchen. Oेbewacht etw. Der große Hund bewacht das ganze Gründstück. Hunde verbeissen sich ineinander Die Hunde haben sich so ineinander verbissen, dass nur die Polizei sie trennen konnte. $-\mathcal{d e r} \mathrm{H}$. hört (,gut erzogen sein) Der Hund bört aber (gut). Oे folgt (,gut erzogen sein)

H. (DAT) + VERB $כ$ dem H. pfeifen Der Halter pfeift seinem Hund, doch er kommt nicht.

H. (AKK.) + VERB Teinen H. halten, den H. anleinen Im Park, muss man Hunde anleinen. den H. an die Leine nehmen, den H. loslassen, כิden H. rufen, כen H. streicheln, den $\mathrm{H}$. auf jmdn. hetzen

PRÄP. „MIT“ $Э$ mit dem H. (raus) gehen Mit einem Hund muss man jeden Abend raus (gehen). $\boldsymbol{\vartheta}$ mit dem H. spazieren gehen, $\boldsymbol{\vartheta}$ mit dem H. spielen $\boldsymbol{\vartheta}$ mit dem H. Gassi gehen

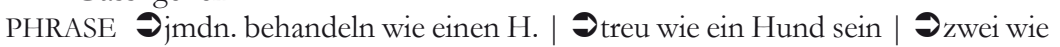
Hund und Katze zwei, die ständig gegenseitig ärgern bzw. überhaupt nicht ausstehen können Sie waren Geschwister aber auch zwei wie Hund und Katze.

Einige der Kollokatoren kommen im Stilduden als Lemmata nicht vor wie zottig, herrenlos, fletschen, hecheln, streunen. Einige der Kollokatoren kommen mit anderen Basiswörtern vor: so struppig im Beispiel struppiges Fell, kläffen (als Lemma) mit Köter, Dackel; spielen kommt mit einem Katzenbeispiel vor. In einigen Fällen ist das Basiswort Hund nicht mit dem adjektivischen Kollokator knurrend verbunden, sondern mit dem Verb knurren, ebenso bei bellen.

In manchen Fällen ist die Kollokation unter dem Adjektiv bzw. dem Verb - oft als Beispielsatz - angeführt: bissiger Hund unter bissig, der Hund frisst unter fressen. Der Hund schnüffelt ist unter schnüffeln aufgeführt, unter wittern wird als Beispielsatz der Hund wittert Wild angegeben, analog unter bewachen der Beispielsatz der Hund bewacht das Haus, analog unter pfeifen der 
Satz der Jäger pfeift seinem Hund, unter halten das Beispiel ich halte mir einen Hund, unter folgen der Satz der Hund folgt mir aufs Wort, unter spazieren die Wendung jmdn. spazieren fübren.

Den Hund rufen wird als Kookkurrenz in unseren Materialien sichtbar, ist aber für ein Wörterbuch zu unspezifisch, um unter rufen entweder als Kollokation oder als Beispielsatz aufgeführt zu werden. Es handelt sich also aus der Sicht der Intuition der BearbeiterInnen um eine unspezifische Kookkurrenz, die in einem Wörterbuch der Kollokationen nicht aufgeführt werden muss. Gassi gehen stellt wohl eine regional- bzw. nationalspezifische Variation dar. Treu kommt weder mit einer Hunde-Wendung noch mit einem Beispielsatz mit Hund vor (sondern in einem Beispielsatz mit Katze).

Laune, die

ADJ. Эgute L. (haben), (in) guter/bester L. (sein) Nach all dem Ärger bin ich nun wieder guter Laune. schlechte L. (haben), Эüble L. (haben), (in) übler/übelster L. sein

SUBST. $\boldsymbol{\nabla} \cdot$ Laune(n) der Natur Schnee im Juni ist eine Laune der Natur.

H. (subj.) + VERB die L. (ver-)bessert sich Mit dem Abendessen warten wir, bis sich deine Laune wieder gebessert hat.

H. (AKK.) + VERB jmdm. die (gute) L. verderben | seine (schlechte) L./seine Launen an jmdm. auslassen Musst du deine Launen immer an deinem kleinen Bruder auslassen? | jmds. (schlechte/miserable/...) Laune ertragen Tagtäglich musste das Kind die schlechte Laune seines Vaters ertragen.

H. (DAT.) + VERB $Э$ die (gute) L. verlieren Wenn man ständig getadelt wird, kann man schnell seine gute Laune verlieren.

PRÄP. „AUS“ Эaus einer Laune heraus (,plötzlich, ohne langes Überlegen') Er entschied sich ganz, spontan, aus einer Laune heraus, zu verreisen.

„BEI“ jmdn. bei L. halten (,jmdn. ablenken, indem man für gute Stimmung sorgt") „UNTER“ unter jmds. L. leiden

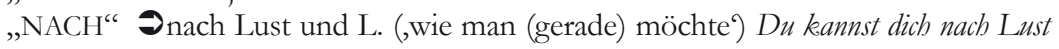
und Laune entscheiden.

PHRASE •Der/die hat (aber) eine Laune!

Die Kollokation gute Laune ist in der Variante gut gelaunt unter dem Adjektiv gut aufgeführt, ebenso analog üble Laune unter übel bzw. schlechte Laune als Beispielsatz unter schlecht; unter jmds. Launen leiden (ohne Relativierung des Plural-n-s) unter leiden. Die Kollokation nach Lust und Laune findet man unter dem Lemma Lust.

\section{Ehre, die}


Beleidigung ist eine Verletzung der persönlichen Ehre eines anderen. $\boldsymbol{\nabla} \cdot$ bürgerliche (fachspr.: ,Anerkennung und Achtung gemäss den Regeln einer Gesellschaft') | Ogekränkte• Hass und gekränkte Ehre seien das Motiv des schrecklichen Mordes. $\boldsymbol{\partial} \cdot$ verletzte | $\boldsymbol{\partial} \bullet$ die (jmdm.) gebührende E. (,angemessene und jmdm. zustehende Anerkennung;) Beim Applaus wurde auch dem Regisseur des Theaterstücks die ihm gebührende Ehre zuteil. $\boldsymbol{\nabla} \cdot$ verdiente Ehre(n) | $\boldsymbol{\partial} \cdot$ zweifelhafte (ironisch: ,Anerkennung oder Auszeichnung, für eine wenig lobenswerte Handlung oder 
Tatsache) Die Auszeichnung als peinlichster Politiker ist eine zweifelhafte Ehre. - $\cdot j m d m$. die letzte E. (erweisen) (,an jmds. Trauerfeier teilnehmen) Tausende erwiesen dem verstorbenen Fussballspieler die letəte Ehre. • militärische (,repräsentatives militärisches (Begrüßungs-)Ritual') Der Staatsgast wird mit militärischen Ehren empfangen.

SUBST. $-2 \cdot R u h m$ und E. (,besondere Anerkennung) Sein Sieg brachte dem Tennisspieler Rubm und Ehre. | ein Mann von E. (,anständiger und vornehmer Herr') - $\odot$ die E. des Mannes (veralt.)

E. (sUBJ.) + VERB • $\boldsymbol{\ni}_{\mathrm{jmdm}}$. gebührt E. (für etw.) (,jmdm. steht eine angemessene Anerkennung zu') $\bullet \bullet$ jmdm. widerfährt E. | die/seine/ihre E. verbietet jmdm. etw. (zu tun)• Kinder zu schlagen, verbietet mir meine Ehre.

E. (DAт.) + VERB seiner (eigenen)/ihrer/... E. etw. schuldig sein• Eine Entschuldigung nach einem Streit bin ich meiner eigenen Ehre schuldig.

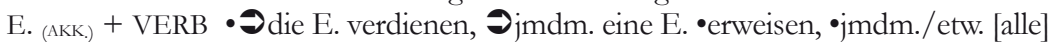
Ehre(n) machen (jmdm./etw. Lob und Anerkennung verschaffen') | jmds. Ehre (be)wahren Durch eine Entschuldigung bei seinen Mitarbeitern konnte der Abteilungsleiter seine Ehre wahren. • jmds. E. verteidigen, Oे seine/jmds. E. retten• Durch zablreiche Ausreden versuchte der Betrüger seine Ehre zu retten. | •jmds. E. verletzen, $\bullet$ jimds. E. beschmutzen Durch die Verfehlungen seiner Tochter sei die Ehre seiner Familie beschmutzt worden. | $\boldsymbol{\nabla} \bullet$ die (besondere/seltene/...) E. genießen, -die E. (miteinander) haben $\mid \bullet$ sich. die E. geben (,sich durch eine Handlung, einen Besuch, eine Aussage selbst Anerkennung und Geltung verschaffen') Der Startenor gab sich die Ehre, in London aufzutreten.

PRÄP. „ZU“ - ••(jmdm.) zu Ehren Es gibt eine Gedenkveranstaltung zu Ehren der Opfer des zpweiten Weltkriegs. | •wieder zu Ehren kommen, $\boldsymbol{\nabla} \cdot$ zu neuen Ehren kommen (,wieder gewürdigt werden), $\boldsymbol{\nabla} \bullet$ etw. zu neuen Ehren verhelfen Die Katæe verhalf Opas altem Sessel zu neuen Ehren, indem sie ibn als Schlafplatz, wählte.

„BEI“ •jmdn. bei der/seiner/... E. packen

„AN" $\boldsymbol{O} \bullet$ jmdn. an der/seiner/... E. packen

„IN“ $\boldsymbol{\partial} \bullet$ etw. in E. halten (,etw. wertschätzen und pflegen) $\mid \boldsymbol{\partial} \cdot$ jmdn. in seiner E. kränken, $\boldsymbol{\vartheta} \bullet$ sich in seiner E. angegriffen fühlen,

„ZUTEIL“ $\boldsymbol{\nabla} \cdot j \mathrm{mdm}$. wird E. zuteil Mit der Auszeichnung für sein Lebenswerk wurde dem Musiker späte Ehre zuteil.

„ZUVIEL“ 2 -zuviel (der) Ehre (Ausdruck der Bescheidenheit) Vielen Dank für die Geschenke, aber das ist zuviel der Ehre.

„MIT“ $\boldsymbol{\vartheta} \bullet$ etw. mit Ehren bestehen (,sehr gut und mit Auszeichnungen abschneiden $) \mid \partial \cdot j m d n$. mit (großen/militärischen/...) Ehren empfangen

„UM““ •jmdn. um seine E. bringen Der Ausrutscher in der letəten Kurve bätte die Eisschnellläuferin fast um ihre Ehre gebracht. $\mid \bullet$ etw. [nur] um der E. willen tun

„AUF“ $\partial \cdot$ ein Angriff auf jmds. E. sein

PHRASE •Es ist/war mir eine E. (Ausdruck zur Erwiederung eines Dankes) Vielen Dank, dass Sie mir die Tür aufgehalten haben - Es war mir eine Ehre. $\mid \cdot$ Was verschafft mir die E.? (höflich bis ironische Frage nach dem Grund für einen

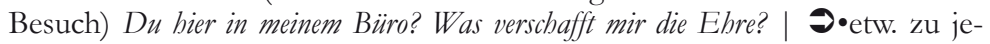
mandes E. sagen $\mid \bullet$ Ehre, wem E. gebührt. $\mid \bullet j$ imds. Wort in Ehren (höflicher Ausdruck zur Einleitung einer Widerrede) Dein Wort in Ehren, aber dieser Vorschlag ist einfach unakezptabel. - • seinem Namen alle E. machen Herr Schlecht macht seinem Namen alle Ebre. 
Hier gibt es keine Kollokatoren, die als Lemmata im Stilduden nicht vorkommen. Hingegen gibt es Kollokatoren mit sehr weiter Bedeutung und großer Häufigkeit wie groß, geben, verdienen oder widerfahren, die kookkurrent mit Ehre vorkommen, aber deren Verbindung mit Ehre weder in einer Kollokationsdarstellung noch als Beispielsatz sichtbar werden. Unter hoch, bürgerlich, kränken, verletżen, Ruhm, erweisen, genießen wird Ehre im Beispielsatz mit dem Lemma verbunden. Verdienen, widerfahren und geben, die als Kookkurrenzen aufscheinen, kommen im Wörterbuch auch als Beispielsätze nicht vor, was bei geben als „Allerweltswort" wenig erstaunlich ist. Im Unterschied $\mathrm{zu}$ den Hund rufen ist jmdm. die Ehre geben auch einfach falsch bzw. Ausweichstrategie bei Nicht-Erinnern des Verbs erneisen. Die präpositionalen Kollokationen zu Ehren kommen bzw. jmdn. bei der Ehre packen sind entweder nur als Beispielsatz (im ersten Beispiel) bzw. gar nicht im Wörterbuch (im zweiten Beispiel) zu finden. Die kollokativen Adjektive groß etc. sind zu unspezifisch, als dass darunter Ehre-Kollokationen - wenn auch als Beispielsätze aufgeführt wären.

Insgesamt ist also ein beachtlicher Teil der in Korpora zu findenden Kookkurrenzen bzw. Kollokationskandidaten in Beispielsätze verpackt bzw. unter anderen als den substantivischen Bestandteilen verzeichnet. Gegen letztere Praxis ist nichts einzuwenden, sie sollte allerdings systematisch und transparent gehandhabt werden. Die Aufführung von Kollokationen in Beispielsätzen ist Ausdruck des Kollokationsempfindens und der Kollokationskenntnis derjenigen, die den Artikel verfasst haben, bzw. kann auch auf die Beleglage zurückgehen, ist aber für diejenigen, die das Wörterbuch benutzen, in keiner Weise transparent. Ein ebenso beachtlicher Teil ist jedoch auch im Duden Stilwörterbuch, das den Kollokationen des Deutschen wohl am besten oder jedenfalls vergleichsweise gut Rechnung trägt, ohne den Begriff explizit zu verwenden bzw. die Kollokationen systematisch lexikografisch aufzuführen, nicht enthalten.

Der Ertrag der Arbeit durch Korporaabfragen ist von der Zusammensetzung, Strukturierung und Größe des Korpus abhängig, sowie von Lemmatisierungsprogrammen und der Berechnung der Kollokationen. Weiter hängt der Ertrag auch davon ab, welchen Stellenwert das abzufragende Element im Wortschatz insgesamt bzw. in den abgefragten Texten hat (vgl. Durĉo i.Dr.).

Kollokationen mit semantisch spezifischen, veraltenden oder veralteten oder unikalen Elementen, also eher seltenen Elementen des Wortschatzes, findet man in der Korpusabfrage leichter - nicht häufiger - als allgemeine Verben oder Adjektive wie legen, stellen, setzen, geben, viel etc. als Teile von Kollokationen. Das ist ein Problem der Bearbeitbarkeit der Ergebnisse einer Kookkurrenzanalyse. Damit diese Verben oder Adjektive etc. als signifikant oben in der (möglicherweise recht langen) Liste erscheinen, müssen sie wirk- 
lich sehr eng mit der Basis verbunden sein und recht häufig in dieser Kombination vorkommen. Sonst stehen sie weiter hinten in der Liste, wo der Aufwand der Unterscheidung von Spreu und Weizen zu groß wird. Präfigierte Verben, die gerade oft mit semantisch wenig spezifischen Wörtern wie stellen, legen, setzen etc. gebildet werden, werden von den Lemmatisierungsprogrammen aber nicht immer richtig aufgelöst. Das heißt, wenn Er stellt ihm ein Bein im Korpus vorkommt, kann es sein, dass das Verb einstellen erkannt wird, genauso aber auch anders herum, dass bei er stellt ihn nach längeren Gesprächen und reiflicher Überlegung letztendlich noch nicht ein nur stellen lemmatisiert wird.

Es ist wichtig und nötig, die Tradition der Wörterbucharbeit miteinzubeziehen, um die Schwächen der Korpusanalyse beim Auffinden bzw. mit Bezug auf die Bearbeitbarkeit der Ergebnisse auszugleichen.

Zu diesen Schwächen der Korporaabfrage gehört, dass sich mit Bezug auf seltene Elemente des Wortschatzes, wie sie gewisse Idiome und eventuell auch Kollokationen bei gleichzeitiger Salienz und Auffälligkeit darstellen, oft einfach keine Suchergebnisse einstellen, obwohl man als kompetenter Sprecher/kompetente Sprecherin weiß bzw. zu wissen meint, dass es die Verbindung gibt und geben muss. In solchen Fällen kann die oft überreichliche und schlecht bzw. gar nicht sortierte Beleglage der Internetabfrage weiterhelfen - wenn es nicht um Phänomene der gesprochenen Sprache, syntaxorientierte Phänomene mit diskontinuierlicher Repräsentation oder ähnliche geht - beides ist bei Internetrecherchen nicht so leicht zu finden. Ein Beispiel dafür: In einem Zeitungstext spricht Kaspar Villiger von einem Beispiel erster Sorte. Die Kompetenzvermutung ist, dass Erster Güte gemeint sein müsste - beides weder im DWDS noch im CHTK. ${ }^{1}$ Die Suche im Internet gibt 521.000 Treffer an (12.02.2010) und enthält sicherlich viel in diesem Zusammenhang Unbrauchbares, jedoch auch in Spitzenstellungen Beispiele vom Typ Diplomatischer Fehltritt erster Güte bzw. ein Multimediamonitor erster Güte, während die Suche nach erster Sorte auf ganz andere Schienen führt, wie die erste und die zweite Sorte.

\subsection{Die Funktionen eines Kollokationenwörterbuches}

Weil Kollokationen semantisch transparent sind, haben entsprechende Wörterbücher weniger eine Funktion für das Verstehen als für das Produzieren. Das ist auch das Anliegen von Hausmann (2004) bzw. seines Momo: Hilfe für den armen Momo, der nicht weiß, wie das richtige Verb zum gewünschten Substantiv lautet. Um die Ausdrucksnot des bedauernswerten Momo zu lindern, müssen alle Kollokationen unter dem Substantiv zu finden sein, wenn Momo vom Substantiv ausgeht.

CHTK $=$ Schweizer Text Korpus, ein Projekt im Rahmen des Digitalen Wörterbuchs der deutschen Sprache. 
Aus der Sicht eines lernenden Sprachbenutzers ist es richtig, dass Substantiv-Verb-Kollokationen unter dem Substantiv aufgeführt werden. Aus der Sicht der Sprachbeschreibung und Sprachreflexion und im Hinblick auf das zu entwickelnde Sprachwissen ist nicht zu vergessen, dass es weitere nichtsubstantivische Kollokationsstrukturen gibt und dass für die Bewusstheit von sprachlichen Einheiten im Allgemeinen und Kollokationen im Besonderen jedes kollokative Element als Ausgangspunkt für das Nachschlagen von Bedeutung ist.

An welches Element man sich nämlich bei gefühlter Eingebundenheit in die Festigkeit von festen Wortverbindungen erinnert, ist nicht vorauszusagen. Es kann durchaus interessant sein, unter stellen nachzuschlagen, welche typischen kollokativen Leistungen das Wort übernimmt, ebenso, wie es interessant sein kann, nachzuschlagen, was man jemandem üblicherweise genau vergällen kann.

Das ist auch für native speakers von Bedeutung, weil sich in journalistischen und anderen Gebrauchstexten sehr viele fragliche Kollokationen bzw. falsch verwendete Kollokationen finden, wonach beispielsweise Hoffnungen auf jemandem lasten. Eine solche Äußerung könnte bei entsprechender Absicht eine aussagekräftige Variante zu Hoffnungen, die auf jmdm. ruhen bzw. eine ebenso aussagekräftige intendierte Kontamination von Hoffnung, die rubt, und Verantwortung, die lastet, darstellen. Analoges gilt für einen Beleg, wonach jmdm. die Teilnahme vergällt wird.

\section{Differenzierung der Sichten vor dem Hintergrund verschiedener lexikografischer Projekte}

Verschiedene Wörterbücher setzen unterschiedliche lexikografische Arbeitsmöglichkeiten voraus. Kollokationen in einem Wörterbuch zum deutschen Grundwortschatz für Sprachlernende der Erst- und Zweitsprache, insbesondere Schülerinnen und Schüler, mit ca. 2.000 Lemmata stellen eine vollkommen andere Aufgabe dar als Kollokationen in einem Dialektwörterbuch des Baseldeutschen mit ca. 10.000 Lemmata oder als Kollokationen in einem Variantenwörterbuch des Deutschen.

Wenn man Kollokationen versteht als nicht oder nur schwach idiomatische Wortverbindungen (vgl. Burger et al. 2007, S. 7), so verlangt dies eine Abgrenzung in zwei Richtungen. Zum einen sind sie von Wortkombinationen abzugrenzen, deren Elemente weder durch Realien, noch durch die Semantik noch durch den Usus überdurchschnittlich prädestiniert sind, miteinander vorzukommen, also von freien Wortverbindungen. Zum anderen sind sie von den übertragenen (idiomatischen) Wortverbindungen (Phrasemen) abzugrenzen, deren Bedeutung sich nicht wörtlich entschlüsseln lässt (beispielsweise starker Tobak, kalter Kaffee). 
Von einer kollokativen Wortverbindung kann man dann sprechen, wenn ein Sachverhalt durch mehrere Wortkombinationen ausgedrückt werden kann, aber von den SprecherInnen/SchreiberInnen häufig ein und dieselbe gewählt wird bzw. beim Sprechen oder Schreiben von fremdsprachigen SprecherInnen auch bei Berücksichtigung der Bedeutungen der Einzelwörter eine unübliche Wortverbindung entsteht, weil sie die kollokative Wortverbindung nicht kennen. Analoges gilt auch in der Erstsprache, wenn es um professionelle oder professionalisierte Ausdrucksweisen geht. Nicht routinisierten Umgang mit Situationen, Laien bzw. Outsidern erkennt man nicht zuletzt an ihrem Sprachgebrauch.

Oft lässt sich bei den Kollokationen eine einseitige, eventuell auch gegenseitige Evozierbarkeit feststellen, also dass ein Ausdruck seinen Kollokationspartner gewissermaßen zwangsläufig fordert, wie es bei den ungleichgewichtigen folgenden Beispielen Abbilfe schaffen oder Amok laufen der Fall ist. Diese Prädisposition, dass für einen bestimmten Sachverhalt häufig die gleiche Wortverbindung aus mehreren möglichen Wortkombinationen ausgewählt wird, lässt sich in Textkorpora mittels der Kookkurrenzanalyse statistisch nachweisen. Wissenschaftlich kann man sich Kollokationen sowohl von sprachsystematischer Seite als auch von pragmatischer Seite nähern. In der angewandten Sprachwissenschaft führt der sprachsystematische Ansatz in seiner konsequenten Umsetzung zur korpusanalytischen Ermittlung von Kookkurrenzen, also solchen Wortpaaren die - ungeachtet der Syntax und Semantik - rein statistisch überdurchschnittlich oft in einem definierten maximalen Abstand (meist fünf Wortzeichen) miteinander vorkommen.

\subsection{Das Kollokationenwörterbuch: Wörterbuch der typischen und gebräuchlichen Wortverbindungen der deutschen Sprache}

Mit dem Wörterbuch der typischen und gebräuchlichen Wortverbindungen der Deutschen Sprache soll eine Lücke in der Deutschen Lerner-Lexikografie geschlossen werden, die beispielsweise im Englischen längst mit dem vielgenutzten „Oxford collocations dictionary for students of English“ (2009), dem „Dictionary of selected collocations“ (1999) und dem „Collins Cobuild English collocations“ (1995) geschlossen wurde, wenngleich das Kollokationenwörterbuch von viel bescheidenerem Umfang als die genannten englischsprachigen Wörterbücher ist.

Kollokationen sind im Zweitspracherwerb noch nach sieben Jahren Unterricht auch in einfachen Texten schwierig, das Können der LernerInnen ist unberechenbarer als beim kindlichen Erstspracherwerb und von der Lehrperson abhängig bzw. von der Übereinstimmung von Erstsprache und unterrichteter Sprache (vgl. Meier 2010). 
Die Kenntnis der Kollokationen einer Sprache ist ein unverzichtbarer Baustein für ein unmarkiertes Sprechen und Schreiben und sollte dementsprechend systematisch in den Sprachunterricht einbezogen werden. Das auf einen Basiswortschatz beschränkte Wörterbuch der typischen und gebräuchlichen Wortverbindungen des Deutschen soll einen systematischeren und reflexiven Umgang mit Kollokationen in Lehr- und Lernmitteln ermöglichen.

Es wird ein korpusbasiertes Kollokationenwörterbuch für die Zielgruppe der fortgeschrittenen Sprachlerner für den Erst- und Zweitsprachunterricht angestrebt. In der Praxis sollte es zu einem nützlichen Nachschlagewerk für Sprachlernende werden, die über den Anfängerstatus hinaus sind, insofern als schon etwas fortgeschritten betrachtet werden können und in Termini des Europäischen Referenzrahmens auf Stufe A2, B1 und B2 anzusiedeln sind.

Das erste Ziel, eine Lemmabasis von 2.000 Wörtern zu erarbeiten, welche als Grund- oder Basiswortschatz den Rahmen des Kollokationenwörterbuchs vorgeben, wurde mit folgenden Quellen und Korpora bearbeitet.

Lern-/Grundwortschätze:

- Lübke, Diethard (2001): Lernwortschatz Deutsch: Deutsch - Englisch. [ca. 3.100 Lemmata];

- Bock, Heiko (Hg.) (1999): Basic German Vocabulary. [ca. 4.000 Lemmata];

- Wiktionary-Grundwortschatz-Liste: http://de.wiktionary.org/wiki/Wiktionary: Projekt:Grundwortschatz_plus_Wiktionary_minimum [ca. 1.400 Lemmata];

- Remanofsky, Ulrich (2000): Zertifikatstraining Deutsch. Wortschatz. [ca. 2.000 Lemmata];

- Schnörch, Ulrich (2002): Der zentrale Wortschatz des Deutschen: Strategien zu seiner Ermittlung, Analyse und lexikografischen Aufarbeitung. Mannheim, Institut für Deutsche Sprache.

Korpora:

- Lemmaliste des Schweizer Text Korpus (CHTK) und des DWDS nach Häufigkeit;

- Wortlisten der häufigsten Wörter des Wortschatzportals Leipzig: http:// wortschatz.uni-leipzig.de/html/wliste.html;

- IDS Mannheim: Korpusbasierte Wortgrundformenliste DeReWo, v30000g-2007-12-31-0.1, mit Benutzerdokumentation, www.ids-mannheim .de/kl/derewo/.

Speziell zur Berücksichtigung der nationalen Varianten wurde bei der Erstellung des Basiswortschatzes das Variantenwörterbuch (Ammon et al. 2004) mit einbezogen und der Basiswortschatz um die nationalen Varianten ergänzt. 
Da sich auch Lehr- und Lernmaterialien an Basiswortschätzen orientieren, wird auf diese Weise zu gewährleisten versucht, dass der Wörterbuchinhalt und der Kenntnisstand von Sprachlernenden möglichst große Übereinstimmungen aufweisen. Es hat sich gezeigt, dass Substantive bei der statistischen Ermittlung des Basiswortschatzes deutlich übergewichtet sind - etwa zwei Drittel der Lemmata sind substantivisch.

Nur eines der mehreren Lemmata der gesuchten Kollokationen muss zum Basiswortschatz gehören, weil Kollokationen oft Basiswortschatzlemmata in Kombination mit sehr allgemeinen und sehr spezifischen, veralteten, eventuell auch unikalen Elementen enthalten. Zudem spielt die Frage der Zugehörigkeit der Bedeutung zum Basiswortschatz eine Rolle: sich etwas nicht nehmen lassen besteht zweifellos aus Elementen des Basiswortschatz, ob auch die Bedeutung dazugehört, ist eine andere Frage, die nicht zuletzt mit Bezug auf die Rolle der Kollokation in Alltagstextsorten der besprochenen Art entschieden werden soll.

Der relevante Basiswortschatz wurde im Redaktionssystem erfasst und als Ausgangsbasis für die Korpusabfragen zur empirischen Datengewinnung verwendet. In der Bearbeitung spielen neben den Kriterien des Basiswortschatzes und der Frequenz die Kriterien der Bedeutung(en) des Lemmas und der darauf aufbauenden Kollokation, sowie das Vorkommen in Textsorten des Alltags, zu dem auch Antragsformulare für die Subventionierung der Krankenkassenprämien, die Steuererklärung sowie RoutineGespräche an Schaltern aller Art gehören, Kommunikationssituationen, die über den routinisierten Charakter hinaus zumindest für die eine der beiden Kommunikationsseiten auch einen professionellen Charakter haben.

Die Kookkurrenzenanalyse von Korpora bringt zu viele nicht wörterbuchrelevante Daten hervor und die rein kognitive und pragmatische Ermittlung der Kollokationen ist neben dem Arbeitsaufwand problematisch hinsichtlich der Repräsentativität des gefundenen Materials. Die Lösung besteht in der Kombination der beiden Verfahren, der Datenerhebung mittels korpusanalytischer Methoden und der anschließenden Auswertung der Daten unter pragmatisch-semantischen Gesichtspunkten.

Ein Beispielartikel rechnen: Es wird versucht, zwischen typischen und gebräuchlichen Wortverbindungen im obigen Sinn zu differenzieren und die Unterscheidung durch fette Initialen (typische Wortverbindungen) bzw. Fettdruck (gebräuchliche Wortverbindungen) visuell sichtbar zu machen. Weiter werden Bedeutungsangaben nur im Fall von polysemen Bedeutungen gemacht. Hingegen werden in jedem Fall auf der Basis empirisch erhobener Textausschnitte möglichst aussagekräftige Beispiele gegeben. 
rechnen

1 eine mathematische Rechnung durchführen

ADV. genau Genau rechnen vor dem Immobilienkauf. I richtig, falsch I schriftlich, im Kopf, mit dem Taschenrechner Einfache Rechnungen lassen sich schneller im Kopf als mit dem Taschenrechner rechnen.

ADV. + R. (PART.) rund Ein Fußballer verdient rund gerechnet 3 Millionen Euro im Jahr.

knapp, hoch Hoch gerechnet bleiben dir von deinem Einkommen noch 30.Franken am Monatsende.

2 „mit etw. / jmdm. rechnen“: planen

ADV. fest Ich habe fest mit deinem Kommen gerechnet. I vorläufig nicht Die Meteorologen rechnen vorläufig nicht mit einer Wetterverbesserung. I frühestens in einer Woche/zwei Tagen/... Mit einer Antwort auf Ihre Bewerbung

\section{3 sparsam sein} können Sie frühestens in einem Monat rechnen.

R. (INF.) + VERB müssen Am Monatsende bleibt ibnen nicht viel Geld, da müssen sie bei jedem Einkauf rechnen. I nicht zu r. brauchen Er verdient genug, dass er nicht bei jedem Restaurantbesuch zu rechnen braucht.

PHRASE mit jedem Cent r. müssen (,wenig Geld (übrig) haben?) Eine Familie mit vier Kindern muss mit jedem Cent rechnen.

\subsection{Das Neue Baseldeutsch Wörterbuch}

Noch einmal andere Fragen stellen sich beim Neuen Baseldeutschen Wörterbuch (vgl. Christoph Merian Stiftung (Hg.) 2010, www.baseldeutsch.ch). Für relativ kleine Dialekte gibt es oft nur ein vorliegendes Wörterbuch, von dem man ausgehen kann. Dazu kommt als Informationsquelle die wertvolle Intuition der Lexikografen, die man weder ausschalten kann noch soll, auf die man sich aber auch nicht beschränken sollte. Deswegen wurden die Baseldeutsch-Sprechenden durch die lokalen Radio- und Zeitungsmedien auf eine Online-Umfrage zum Baseldeutsch-Wörterbuch aufmerksam gemacht, in deren Kontext sie Sprachgebrauchsdaten zu schlecht belegten Wörtern beisteuern konnten. Sie konnten Angaben machen zur persönlichen Gebräuchlichkeit und Kenntnis sowie typische Kontexte angeben. Die folgenden Daten beziehen sich auf die Ergebnisse dieser Umfrage.

Mit Korpora ist es bei Dialekten insofern schwieriger, als zwar Einiges an Texten schriftlich verfügbar ist, die Texte jedoch großmehrheitlich nicht digitalisiert und in vielen Fällen (bei Theaterstücken für Laiengruppen etc.) auch nicht gedruckt und nur als Schreibmaschinen-Manuskript vorhanden sind. Dazu kommt, dass die nicht normierte Dialektschreibung, auch wenn die Texte digitalisiert werden, die elektronische Suche nicht erleichtert. Dialektwörterbücher, die sich an ein Laienpublikum richten und regional vermarktet werden, haben zudem eine Tendenz, sich rückwärtsgewandt auszurichten. Laien suchen in einem kleinräumig ausgerichteten Dialektwörterbuch 
ein Nachschlagewerk zu sprachlichen Fragen der regionalen Identität. Regionale Identität ergibt sich in Zeiten, in denen Kleinräumigkeit durch tendenzielle Globalisierung bedroht ist, in viel stärkerem Ausmaß durch die Geschichte als durch ungewisse Zukunftsfunktionen oder die Modernität eines Dialekts, für die Kriterien und damit die Erkennbarkeit fehlen. Zudem gilt eine beschränkte Auswahl historischer Sprachformen, die man sich als Schibboleth merken kann, die man vielleicht noch vom Hören oder sogar vom Hörensagen kennt, als interessant. Als Alternative der Datenerhebung wurden hier Fragen an den Sprachgebrauch in einem Internetportal gestellt.

Das Baseldeutsche Wörterbuch wird daher nicht mehr aktuelle Wortformen, die einmal typisch für das Baseldeutsche waren, bei voller deskriptiver Ausrichtung nicht einfach entfernen, sondern als historische Formen, die im Sprachwissen und in der Sprachbewusstheit angesiedelt werden können, kennzeichnen. Es stellt sich sogar die Frage, ob man historische Schreibweisen, die zu ihrer Zeit die Aussprache abgebildet haben und heute noch gut bekannt und geschätzt sind, als orthographische Schreibweisen aufnehmen soll, obwohl sie nicht mehr gesprochen werden und in der Dialektschreibung normalerweise ein aktuelles Transkriptionsprinzip gilt. So beispielsweise mit Bezug auf die Entrundung: Historisch gesehen wurde im Baseldeutschen ein -ö- entrundet als -e- ausgesprochen sowie ein -ü- entrundet als -i- realisiert. Da diese historische Aussprache das höhere Prestige hatte und teilweise noch hat, wurde die entsprechende Schreibweise für die ebenfalls prestigeträchtige und - wie in einer protestantischen Region üblich - streng reglementierte Fasnacht bzw. Fasnachtsschreibung vorschriftsmäßig verwendet.

Das baseldeutsche Adjektiv scheen (hdt.: schön) gilt vielen SprecherInnen und SchreiberInnen heute weniger als artikulatorische Abbildung, die sie nicht mehr ist, sondern als Rechtschreibnorm. Jedoch wird die Entrundung in keiner Weise systematisch gesprochen und geschrieben, sondern nur mit Bezug auf wichtige Fasnachtswörter fallweise realisiert: Niemandem würde es daher einfallen, analog Bürger als Bïrger zu schreiben.

Es gibt also bei der Aktualisierung eines Wörterbuchs viele Fragen zum Umgang mit dem bisher erfassten lexikografischen Material und seiner aktuellen Beurteilung hinsichtlich Bekanntheit und Gebräuchlichkeit, die normalerweise durch ein großes strukturiertes Korpus angegangen werden. Die folgenden Beispiele sollen das illustrieren.

E zrfrìdeni Sach (hdt.: *Eine zufriedene Sache, jedoch auf Hochdeutsch nicht phraseologisch und (daher) ohne die Bedeutung ,eine gute Sache, mit der man zufrieden sein kann"). Eingebettet in einen Kontext wurde der Ausdruck in der Online-Umfrage beispielsweise folgendermaßen gebraucht:

$$
\begin{aligned}
& \text { a. Am Sùnntig usschloofe find ich e zrrìdeni Sach (hdt.: „Am Sonntag } \\
& \text { ausschlafen finde ich *eine zufriedene Sache }{ }^{*} \text { ). }
\end{aligned}
$$


b. Die Fè̀rie sinn e afrì̀deni Sach gsì̀ (hdt.: „Die Ferien waren *eine zufriedene Sache ${ }^{6}$ ).

E glùngene Thüp (hdt. wörtlich: *Ein gelungener Typ, im Hochdeutschen nicht gebräuchlich, ohne die baseldeutsche Bedeutung „ein spezieller Typ“). Der Ausdruck war zehnmal in der Online-Umfrage, wurde neunmal bewertet und von allen neun Personen mit ,schon gehört und weiß, was es bedeutet" gekennzeichnet. Vier Personen gebrauchten den Ausdruck „manchmal“, zwei „oft“, eine „nie“. Eine Person betitelte den Ausdruck als „altmodisch“.

Interessant an dieser Auswahl von Beispielsätzen ist die Einbettung des Ausdrucks hinsichtlich der Palette von Partikeln, die im Kontext des Ausdrucks oft gebraucht werden wie balt e biæ, no, äfach, denn, die in diesen Beispielsätzen von Laien sehr gut die typische sprachliche Vorsicht bei Qualifizierungen von Mitmenschen zum Ausdruck bringen.

(2) a. Wäisch är isch halt e bize glìngene Thüp und nit wie alli andere (hdt. wörtlich: „Weißt Du, er ist halt ein bisschen *ein gelungener Typ und nicht wie alle anderen").

b. Der Feelix isch nòn e glìngene Thüp (hdt.: „Der Felix ist noch *ein gelungener Typ").

c. Das isch äfach e glìngene Tbüp (hdt.: „Das ist einfach *ein gelungener Typ").

d. Dasch mer nòn e glùngene Thüp (hdt.: „Das ist mir noch *ein gelungener Typ").

e. Kènnsch der Walti? Das isch dènn e glìngene Thüp (hdt.: „Kennst Du den Walter? Das ist vielleicht *ein gelungener Typ").

f. Är isch biz e glìngene Tbüp (hdt.: „Er ist ein bisschen *ein gelungener Typ").

Nit gan₹, bache sii (hdt.: *nicht gan₹,gebacken sein im Sinne von „nicht ganz normal sein"). Der Ausdruck war siebenmal in der Umfrage und wurde fünfmal mit „schon gehört und weiß, was es bedeutet“ bewertet. Eine Person verwendete den Ausdruck „manchmal“, zwei „oft“.

Die Beispielsätze bringen im ersten Fall die Semantik und Pragmatik von nit gan₹ bache sii wiederum gut zum Ausdruck, im ersten Fall durch die syntagmatische Anfügung mit seiner Bieridee, im zweiten und dritten Fall durch die Verwendung in der Anrede als ,mäßig schlimme“ Beschimpfung.

a. Dä isch dòch nit gan₹ bache mit sine Bieridee'e (hdt.: „Der ist doch * nicht gan ₹ gebacken mit seinen Bierideen").

b. Du bisch dòch nit ganz bache (hdt.: „Du bist doch *nicht gan₹ gebacken" ${ }^{\prime \prime}$ ).

c. Bisch nit ganz bache, bi dääre Kélti nùr im Pulloower ùff $d$ Strooss z.goo? (hdt.: „Bist Du *nicht gan₹ gebacken, bei der Kälte nur im Pullover auf die Strasse zu gehen?"). 
Öpperem äini bache (hdt.: *Jemandem eine backen im Sinne von „jmdm. eine runterhauen"). Der Ausdruck war zwölfmal in der Umfrage, wurde achtmal bewertet, siebenmal mit "schon gehört und weiß, was es bedeutet“, einmal mit „noch nie gehört“. Der Ausdruck wurde dreimal als „oft gebraucht", zweimal als „manchmal gebraucht", einmal als „,nie gebraucht" taxiert. Als Beispielsätze wurden die prototypische konditionale Verwendung als Rede über Dritte und die Drohung gegenüber einem nervenden Gegenüber angegeben:

Interessant ist, dass die Beispielsätze wie im Fall der obigen Drohung oft - bis auf einen Partikel oder ähnlich - identisch vorgeschlagen werden und dementsprechend in der aufgeführten Verwendung sehr gebräuchlich sein müssen.

a. I bät em ä̈ni könne bache (hdt.: „Ich hätte ihm eine runterhauen können“ im Sinne von „mögen“ oder „wollen“).

b. Däre blöode Kue söt men emòl äni bache (hdt.: „Dieser blöden Kuh sollte man mal eine runterhauen").

c. Wènn d jètž nit sòfort üffhö̈rsch, dènn bach der äini (hdt.: ,Wenn du jetzt nicht sofort aufhörst, haue ich dir eine runter").

Ùff äi Dätsch (hdt.: ,,auf einen Schlag“o). Der Ausdruck war elfmal in der Umfrage, wurde zehnmal bewertet und neunmal mit ,schon gehört und weiß, was es bedeutet“ beurteilt. Eine Person meinte, der Ausdruck wäre „,veraltet“. Drei Personen verwendeten den Ausdruck „oft“, vier „manchmal“.
a. Si bän ùff äi Dätsch drei Kinder bikoo: Drilling! (hdt.: „Sie haben auf einen Schlag drei Kinder bekommen: Drillinge!").
b. Üff äi Dätsch sinn alli wach gsì (hdt.: „Auf einen Schlag waren alle wach").
c. Si hèt ùff äi Dätsch 10000 Frangge gwùnne (hdt.: „Sie hat auf einen Schlag 10.000 Franken gewonnen").
d. Es kìnnt alles ìff äi Dätsch (hdt.: „Es kommt alles auf einen Schlag").
e. Sisch alles îff äi Dätsch koo (hdt.: „Es ist alles auf einen Schlag gekommen").

\section{Fazit}

Insgesamt kann festgehalten werden: Erste Arbeiten an einer separaten Lexikografie der Kollokationen zeigen, dass systematische Korpusanalysen viel zusätzliches Material zu Tage fördern. Es stellt sich die Frage, wie dieses Material aufbereitet werden soll bzw. für wen welche Kenntnisse über Kollokationen nützlich sind. Kollokationen sind grundsätzlich nicht nur im Hin- 
blick auf die Förderung der Sprachrichtigkeit lexikografisch zu erfassen, sondern auch im Hinblick auf Sprachwissen zur Ökonomie der Sprachentwicklung und Sprachwissen zur Musterbildung und zum Mustererwerb wissenschaftlich zu beschreiben und lexikografisch zu verzeichnen.

Angesichts der großen Zahl der auffindbaren Kollokationen ist zu differenzieren und zu gewichten: a) Es steht lexikografisch zunächst die Unterstützung bei der Sprachproduktion im Vordergrund sowie b) der Grundwortschatz bzw. Basiswortschatz, und es sind c) die Unterscheidung von typischen Wortverbindungen und gebräuchlichen Wortverbindungen (den Hund loslassen vs. den Hund anleinen/ an die Leine nehmen) vorzunehmen.

Bei der Dialektlexikografie (mit vergleichsweise kleinen Korpora im Hintergrund) findet man auch zusätzliche Kollokationen, jedoch quantitativ weniger ausgeprägt. Hier sind ergänzende Datenerhebungen über Umfragen hilfreich und liegen die Unterschiede zum Bisherigen vor allem auch in der Artikelstruktur, in der Herausnahme der Kollokationen aus dem inadäquaten Beispielsatzformat und in den Aktualisierungen der Form.

\section{Literatur}

\section{Wörterbücher}

Ammon, Ulrich et al. (2004): Variantenwörterbuch des Deutschen: die Standardsprache in Österreich, der Schweiz und Deutschland sowie in Liechtenstein, Luxemburg, Ostbelgien und Südtirol. Berlin/New York.

Bock, Heiko (Hg.) (1999): Basic German vocabulary. Überarb. Aufl. Berlin/München.

Christoph Merian Stiftung (Hg.) (2010): Neues Baseldeutsch Wörterbuch. Basel.

Collins Cobuild English collocations (1995) = Sinclair, John et al. (Hg.) (1995): Collins Cobuild English collocations on CD-ROM: a comprehensive database of common word patterns from the Bank of English. London.

Dictionary of selected collocations (1999) = Hill, Jimmie/Lewis, Michael (Hg.): Dictionary of selected collocations. Hove.

Duden (2010): Duden. Bd. 2: Das Stilwörterbuch: Grundlegend für gutes Deutsch. 9., völlig neu bearb. Aufl. Hrsg. v. Wissenschaftlichen Rat der Dudenredaktion. Mannheim u.a.

DWDS: Das digitale Wörterbuch der deutschen Sprache des 20. Jahrhunderts. Internet: www.dwds.de (Stand: 24.01.2011).

Lübke, Diethard (2001): Lernwortschatz Deutsch: Deutsch - Englisch: [Wortschatz für das neue Zertifikat Deutsch]. Ismaning.

Oxford collocations dictionary for students of English (2009). Hrsg. v. Colin McIntosh et al. 2. Aufl. Oxford u.a. 
Remanofsky, Ulrich (2000): Wortschatz: überarbeitet für das neue Zertifikat Deutsch. 3., bearb. u. erg. Aufl. Ismaning.

Schnörch, Ulrich (2002): Der zentrale Wortschatz des Deutschen: Strategien zu seiner Ermittlung, Analyse und lexikografischen Aufarbeitung. (= Studien zur Deutschen Sprache 26). Tübingen.

WDG $=$ Wörterbuch der deutschen Gegenwartssprache (1961-1977). Hrsg. v. Ruth Klappenbach und Wolfgang Steinitz. 6 Bde. Berlin.

Wiktionary-Grundwortschatz-Liste: http://de.wiktionary.org/wiki/Wiktionary:Projekt:Grund wortschatz_plus_Wiktionary_minimum (Stand: 16.03.2010)

\section{Sekundärliteratur}

Barz, Irmhild (2007): Wortbildung und Phraseologie. In: Burger et al. (Hg.), S. 27-36.

Bürki, Andreas (i.Dr.): Lexis that rings a bell: on the influence of auditory support in vocabulary acquisition. In: International Journal of Applied Linguistics.

Burger, Harald (2010): Phraseologie: eine Einführung am Beispiel des Deutschen. 4., neu bearb. Aufl. (= Grundlagen der Germanistik 36). Berlin.

Burger, Harald et al. (2007): Phraseologie: Objektbereich, Terminologie und Forschungsschwerpunkte. In: Burger, Harald et al. (Hg.), S. 1-10.

Burger, Harald et al. (Hg.) (2007): Phraseologie: ein internationales Handbuch zeitgenössischer Forschung. (= Handbücher zur Sprach- und Kommunikationswissenschaft (HSK) 28, Teilbd. I). Berlin u.a.

Durĉ́, Peter (i.Dr.): Extracting data from corpora statistically - pros and cons.

Eichinger, Ludwig M. (2004): Von Köpfen, Nägeln und anderen guten Bekannten. In: Steyer (Hg.), S. 1-18.

Filatkina, Natalia/Hanauska, Monika (i.Dr.): Wissensstrukturierung und Wissensvermittlung durch Routineformeln: Am Beispiel ausgewählter althochdeutscher Texte. In: Yearbook der europäischen Gesellschaft für Phraseologie.

Häcki Buhofer, Annelies (1997): Phraseologismen im Spracherwerb. In: Wimmer, Rainer/Berens, Franz Josef (Hg.): Wortbildung und Phraseologie. (= Studien zur deutschen Sprache 9). Tübingen, S. 209-232.

Hausmann, Franz Josef (2004): Was sind eigentlich Kollokationen? In: Steyer (Hg.), S. 309-334.

Hausmann, Franz Josef (1989): Le dictionnaire de collocations. In: Hausmann, Franz Josef et al. (Hg.): Dictionaries. An International Encyclopedia of Lexicography. (= Handbücher zur Sprach- und Kommunikationswissenschaft (HSK) 5). Berlin u.a., S. 1010-1019.

Meier, Stefanie (2010): Kollokationen im Zweitspracherwerb. Masterarb. Basel.

Polenz, Peter von (2009): Geschichte der deutschen Sprache. 10., völlig neu bearb. Aufl. Berlin u.a. 
Steyer, Kathrin (Hg.) (2004): Wortverbindungen - mehr oder weniger fest. (= Jahrbuch des Instituts für Deutsche Sprache 2003). Berlin u.a.

Steyer, Kathrin/Brunner, Annelen (2009): Das UWV-Analysemodell. Eine korpusgesteuerte Methode zur linguistischen Systematisierung von Wortverbindungen. (= OPAL 1/2009). Mannheim. Internet: http://pub.ids-mannheim.de/laufend/opal/pdf/ opal2009-1.pdf (Stand: 24.01.2011).

Steyer, Kathrin (2008): Kollokationen in deutschen Wörterbüchern und in der deutschen Wörterbuchforschung. In: Lexicographica 24. Berlin/New York, S. 185-207. 
